\title{
Distribution ratio of dissolved and particulate REE in surface coastal seawater
}

\author{
Fu FengFu, ${ }^{1}$ Kazunori Shinotsuka, ${ }^{2}$ Mitsuru Ebihara ${ }^{2}$ and Tasuku AKagi ${ }^{1}$ \\ ${ }^{1}$ Faculty of Agriculture, Tokyo University of Agriculture and Technology, Saiwai-cho, Fuchu, Tokyo 183, Japan \\ ${ }^{2}$ Faculty of Science, Tokyo Metropolitan University, Minamiosawa, Tokyo 192-03, Japan
}

(Received March 15, 1996; Accepted February 21, 1997)

\begin{abstract}
Dissolved and particulate forms of rare earth elements (REE) in the surface seawater of coastal area were separately determined with Inductively Coupled Plasma ion source Mass Spectrometry (ICP-MS) in order to discuss the REE partitioning between them. Two different samples were prepared on the occasion of each sampling: one sample was acidified before it was filtrated (AF) and another sample was acidified after filtrated (FA). On the two samples (AF and FA) we measured the REE abundances of the filtrate and the collected matter on the filter separately. In either sample, the filtrate showed a "W type tetrad effect"like variation in the chondrite normalized REE pattern. The partitioning pattern of sample FA shows a clearer "W type tetrad effect"-like variation than that of sample AF, indicating that "labile" showed a "M type tetrad effect"-like variation.

Besides Ce anomaly, anomalous behavior of $\mathrm{Tb}$ has been observed in the filtrate of the sample FA. Tb in the collected shows rather capricious behavior.
\end{abstract}

\section{INTRODUCTION}

In the studies of liquid-liquid extraction for REE, when the concentration ratios between the aqueous phase and the organic phase are plotted in logarithmic scale against the atomic number, four curves, each of which consists of four contiguous elements [La-Ce-Pr-Nd, (Pm)-Sm-Eu-Gd, Gd-Tb-Dy-Ho, Er-Tm-Yb-Lu], are sometimes observed (e.g., Peppard et al., 1969). The effect causing the variation is called the tetrad effect and later a theoretical interpretation was given to the effect by Jørgensen (1970) and Kawabe (1992), based on repulsion energy of $4 \mathrm{f}$ electrons. Masuda and Ikeuchi (1979) determined REE in seawater and pointed out that the tetrad effect was observed in seawater. REE patterns of seawater have been reported by several researchers (Elderfield and Greaves, 1982; Klinkhammer et al., 1983; De Baar et al., 1985a, b; Masuda et al., 1987; Piepgras and Jacobsen, 1992), and Akagi et al. (1993) suggested from the compilation of them that their chondrite normalized REE patterns show the tetrad effect variation, irrespective of analytical methods by which they were obtained. It has not, however, been widely accepted that the pattern of seawater is the result of the tetrad effect, and McLennan (1994) pointed out the possibility of analytical artefact.

There are two major difficulties to prove the effect in nature. One is the analytical difficulty. REE concentrations of natural samples are very low and the ranges of analytical instruments for the determination of trace metals are not accurate enough to detect the effect in question. The other difficulty is to identify the two conjugate phases. If a sample shows a "W type tetrad effect"-like variation, it is usually difficult to identify the other phase which has given rise to the variation in the sample as a result of the interaction. From the empirical results (e.g., Masuda et al., 1987), we can assume that REE pattern shows the "W" type tetrad effect in aqueous phase and the "M" type tetrad effect in solid phase in nature. In order to examine whether REE pattern in seawater shows tetrad effect, the comparison of these two phases 
is considered to be one of the most direct methods. In this paper, we determined REE concentrations in seawaters and in suspended particles in ocean and compared them.

\section{EXPERIMENTS}

\section{Seawater samples}

We sampled surface seawater offshore from Aburatsubo Bay, Misaki, in May, 1994 and in April, 1995. A portion of samples was acidified to $\mathrm{pH}=1$ with $\mathrm{HCl}$ immediately after sampling and was filtrated with a nuclepore filter (pore size $0.4 \mu \mathrm{m}$ ) after more than one month (this sample is called sample AF). Another portion of sample was filtrated immediately after sampling and was acidified to $\mathrm{pH}=1$ with $\mathrm{HCl}$ (this sample is called sample FA). Difference between the samples FA and AF is shown schematically in Fig. 1. In the sample FA, "labile" REE is recovered as "collected", while it is recovered as "filtrate" in the sample AF. The filters used were stored for the determination of REE in suspended particles. The weight of particulate matter collected on the filter was determined as the weight difference between a new filter and the filter on which particulate matter was collected. The filters were dried at $100^{\circ} \mathrm{C}$ before weighing. Each seawater sample was kept in a cleaned bottle.

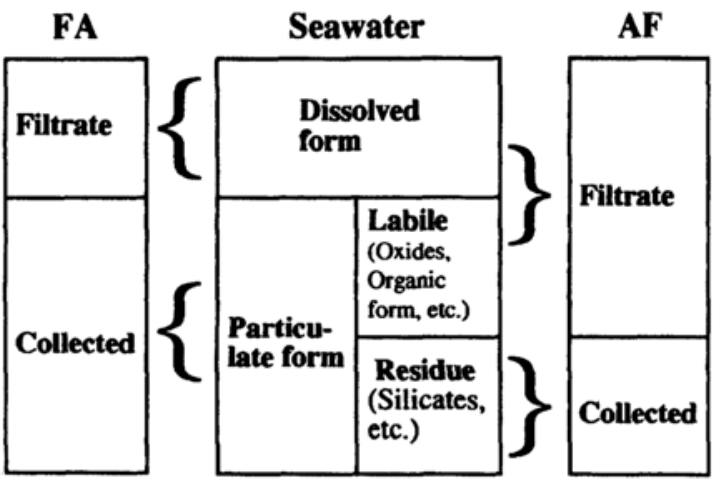

Fig. 1. Schematic diagram showing possible correspondence of samples (FA and AF) to fractions of seawater and particles.

\section{Analytical method}

The analytical method for seawater was based on that developed by Shabani et al. (1992). REE in 1 litter of seawater was preconcentrated by a $\mathrm{C}_{18}$ cartridge loaded with a mixture of HDEHP and $\mathrm{H}_{2} \mathrm{MEHP}$. REE collected on the cartridge was eluted by $40 \mathrm{ml}$ of $6 \mathrm{M} \mathrm{HCl}$. The $6 \mathrm{M} \mathrm{HCl}$ solution was collected in a Teflon beaker and a known amount of In standard solution was added to the solution. The solution was evaporated to dryness and the residue was dissolved again with about 5 $\mathrm{ml}$ of $0.4 \mathrm{M} \mathrm{HNO}$, and the $5 \mathrm{ml}$ solutions was used for the ICP-MS determination with In as an internal standard.

Particulate matters (about $0.015 \mathrm{~g}$ ) collected on filters from seawater (approximately 4 liters) were first boiled with $\mathrm{HClO}_{4}$ and $\mathrm{HNO}_{3}$ with the filters in a Teflon beaker. After complete decomposition, $0.5 \mathrm{ml}$ of HF and a known amount of In standard solution were added and the acids were removed by heating the beaker gently. Finally the residue was dissolved with about $4 \mathrm{ml}$ of $0.4 \mathrm{M}$ $\mathrm{HNO}_{3}$ and the solution was measured with ICPMS for the determination of REE again with In as an internal standard. The blank and recovery were measured in each analysis of seawater and particles. For the recovery tests, about $20 \mathrm{ng}$ of each REE was added to the same samples ("filtrate" and "collected") prior to the analytical procedures. In the ICP-MS measurement, oxides of lighter elements were corrected using oxide forming factors, which were determined each day using several combined standard solutions. The corrections were as much as $20 \%$ at $\mathrm{Gd}-157$ $\left({ }^{141} \mathrm{PrO}\right)$ at maximum, but have never exceeded $10 \%$ of the observed counts for any other elements except Gd.

All the acids used were purified by subboiling distillation and the water used was Milli-Q water. The mixture of HDEHP and $\mathrm{H}_{2}$ MEHP from Kanto Chemical Co., Ltd. was used as it was supplied. The standard solutions of each REE were prepared by dissolving their pure oxides.

The recovery, blank counts and concentrations corresponding to the counts are shown in Table 1 for both the analyses of the filtrate and collected on filters. 
Table 1. Recovery and blank of the analyses

\begin{tabular}{|c|c|c|c|c|c|c|}
\hline \multirow[t]{3}{*}{ Element (Mass number)* } & \multicolumn{3}{|c|}{ Filtrate } & \multicolumn{3}{|c|}{ Collected } \\
\hline & \multirow{2}{*}{$\begin{array}{c}\text { Recovery } \\
\text { (\%) }\end{array}$} & \multicolumn{2}{|c|}{ Blank } & \multirow{2}{*}{$\begin{array}{c}\text { Recovery } \\
\text { (\%) }\end{array}$} & \multicolumn{2}{|c|}{ Blank } \\
\hline & & Counts & Concn.** & & Counts & Concn.** \\
\hline La (139) & 95 & 212 & 0.064 & 97 & 324 & 0.031 \\
\hline $\mathrm{Ce}(140)$ & 96 & 992 & 0.348 & 103 & 1678 & 0.184 \\
\hline $\operatorname{Pr}(141)$ & 96 & 80 & 0.025 & 97 & 110 & 0.010 \\
\hline Nd (146) & 95 & 61 & 0.105 & 97 & 73 & 0.038 \\
\hline $\mathrm{Sm}$ (147) & 96 & 49 & 0.102 & 97 & 45 & 0.028 \\
\hline Eu (151) & 96 & 46 & 0.031 & 97 & 53 & 0.011 \\
\hline Gd (157) & 95 & 48 & 0.091 & 96 & 48 & 0.027 \\
\hline $\mathrm{Tb}(159)$ & 95 & 86 & 0.028 & 96 & 274 & 0.027 \\
\hline Dy (162) & 95 & 55 & 0.071 & 95 & 44 & 0.017 \\
\hline Ho (165) & 96 & 66 & 0.022 & 96 & 61 & 0.006 \\
\hline $\operatorname{Er}(166)$ & 96 & 56 & 0.060 & 95 & 44 & 0.014 \\
\hline $\operatorname{Tm}(169)$ & 97 & 56 & 0.019 & 96 & 55 & 0.006 \\
\hline $\mathrm{Yb}(174)$ & 100 & 73 & 0.082 & 95 & 64 & 0.021 \\
\hline Lu (175) & 100 & 78 & 0.030 & 95 & 71 & 0.008 \\
\hline
\end{tabular}

*In the parentheses are mass numbers at which blank counts were measured.

**The concentration of REE corresponding to the blank counts (pg/l g seawater).

\section{RESUltS AND DISCUSSION}

REE concentrations of seawater and suspended particles

Analytical results are summarized in Table 2. Table 2 shows that the concentrations of REE in sample AF are higher than that in sample FA in seawater, and that the difference is larger for LREE. This is because the HREE are preferentially retained in solution, whereas LREE are adsorbed to surfaces of particles, from which REE were dissolved by acids in the analysis of the sample AF (Koeppenkastrop et al., 1991; Koeppenkastrop and De Carlo, 1993). Also from Table 2 and the sample weights, it is calculated that about $75 \%$ of LREE and $90 \%$ of HREE exist as dissolved form.

Chondrite normalized patterns of seawaters and suspended particles

In this study, we used a set of normalizing values by Masuda et al. (1973) and Masuda (1975), and the chondrite normalized REE patterns of seawaters and suspended particles are shown in Figs. 2 and 3, respectively. Error bar of each point represents $1 \sigma$ deviation, calculated from the results of two analyses shown in Table 2 (1 analysis consists of three measurements) except the collected and filtrate of sample AF in 1995, in which the averages were calculated from two analyses and the larger deviations between the two analyses were selected. The two patterns of the filtrate of FA (1994 and 1995) are similar to each other and those of AF are almost identical, indicating that our analysis was reproducible.

The REE patterns of filtrate of AF are typical one of surface seawater and those of particulate of AF are similar to those of common basalts. From Fig. 2, we can find that REE patterns of the filtrate of sample FA and AF are quite similar to each other, but apparently different at $\mathrm{Ce}$ and $\mathrm{Tb}$ and in the slope of HREE region. Negative Ce anomalies are more greatly seen in sample FA than in sample AF. A positive $\mathrm{Tb}$ anomaly can be seen in REE patterns of the filtrate of sample FA. From Fig. 3, the REE pattern of the collected of sample $\mathrm{AF}$ and FA is very similar to each other except $\mathrm{Ce}, \mathrm{Tb}$ and the slope of HREE region which is slightly smaller in sample AF.

Most of geological samples including rocks and minerals show rather simple chondrite normalized 


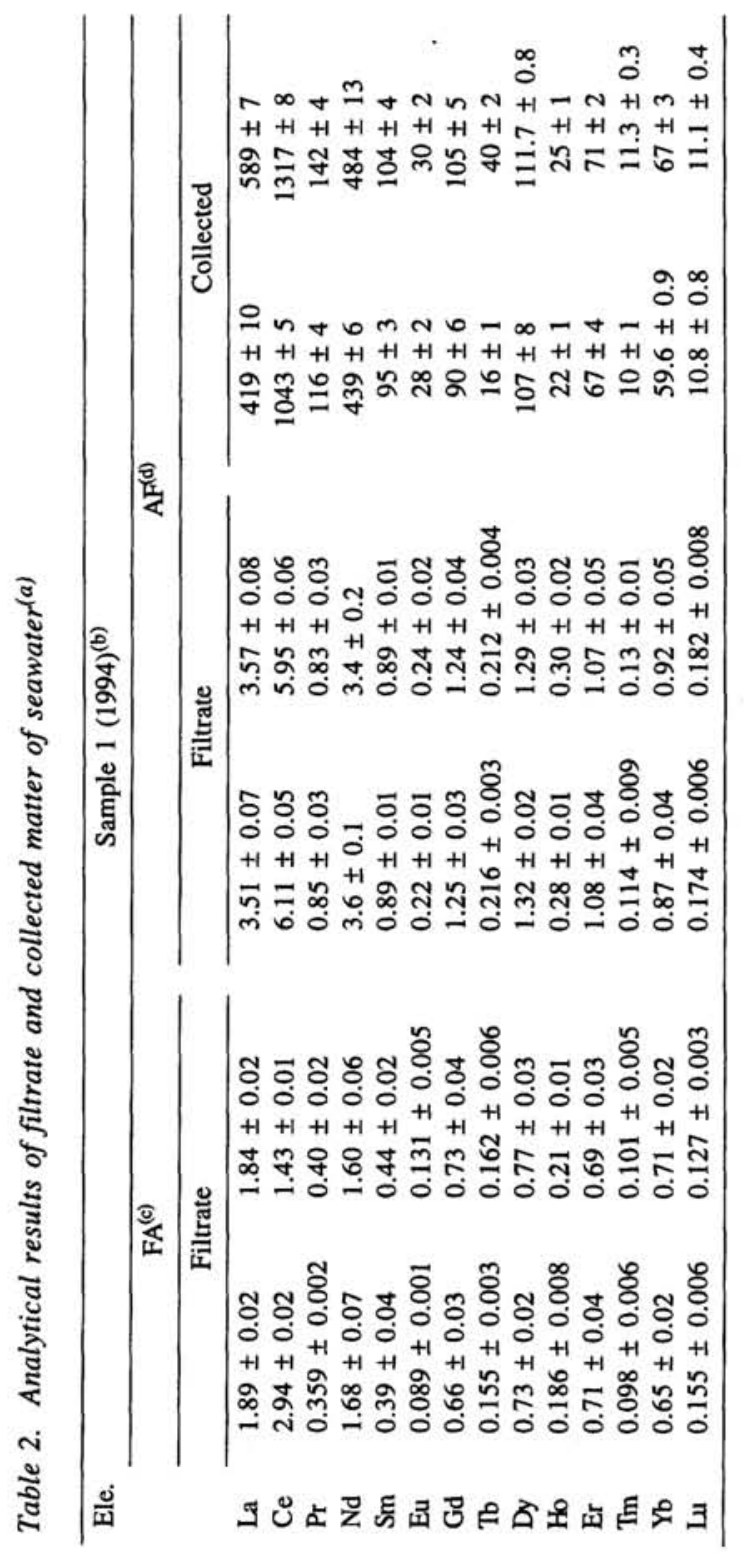




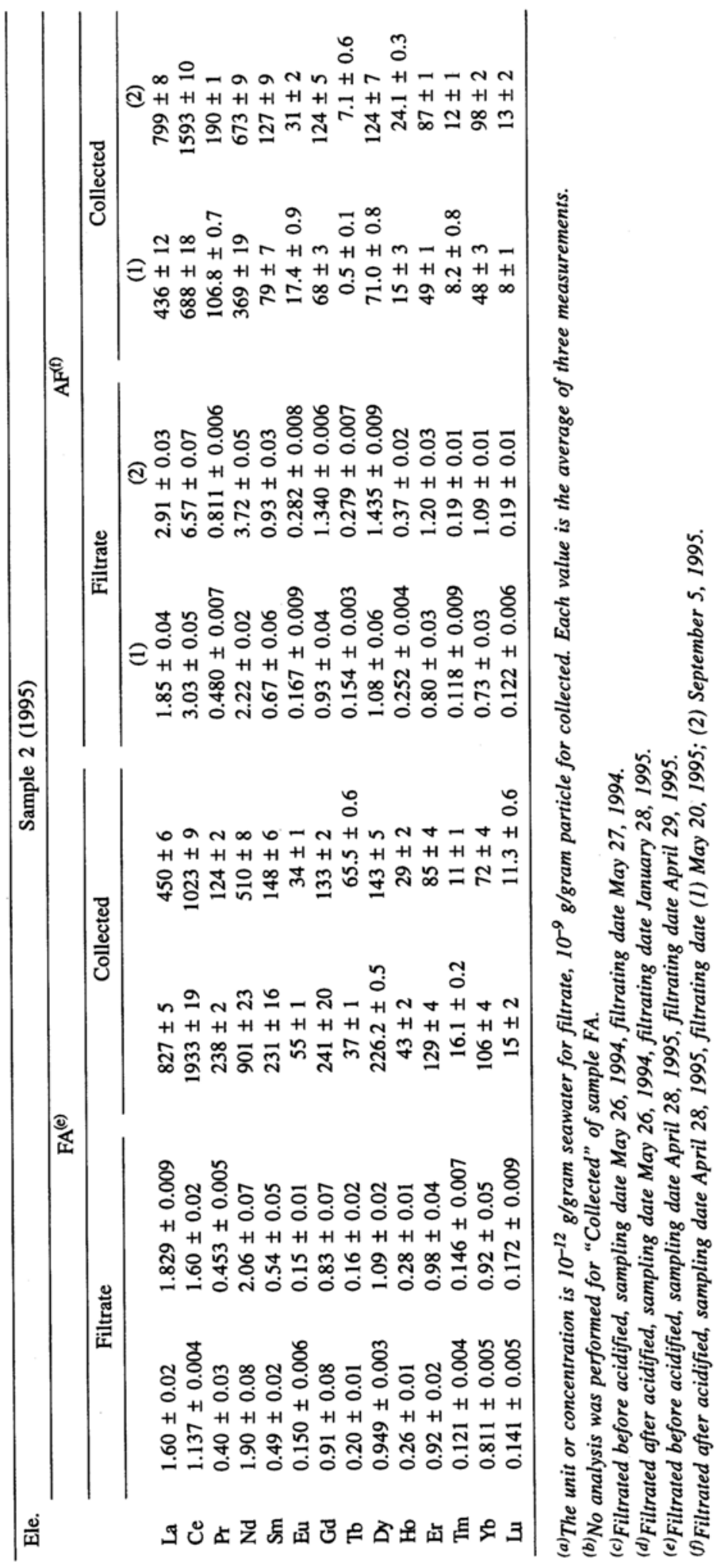



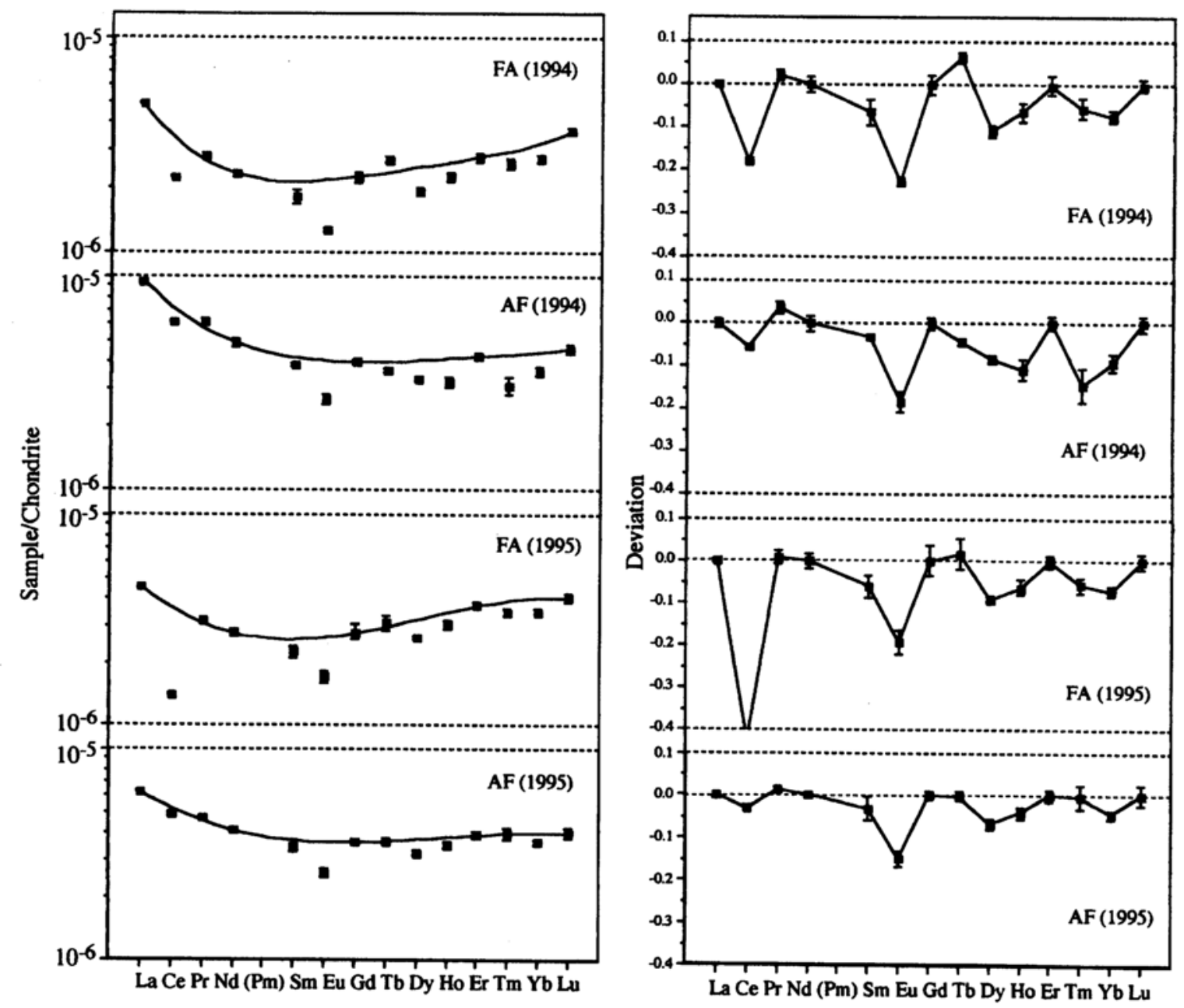

Fig. 2. Chondrite normalized patterns of dissolved REE (the left row) and deviations from fitted smooth curves (the right row), which are determined using a fourth-degree polynomial with respect to the atomic numbers of $R E E$ for the La, Nd, Gd, Er and Lu data points. Deviation = log(normalized measured value/value calculated from the fitted curve). Error bars stand for $1 \sigma$ of six data from two replicated analyses.

patterns (e.g., Henderson, 1984). The REE patterns of the filtrate and collected, however, do not seem to be very simple. We have introduced a background pattern to separate aberrations due to the tetrad effect from the overall variations. In Figs. 2, 3 and 4, the right row of the figures are deviations from calculated curve fitted to normalized values of $\mathrm{La}, \mathrm{Nd}, \mathrm{Gd}, \mathrm{Er}$ and $\mathrm{Lu}$; the five elements were chosen to extract the tetrad effect variation since they are the ends or the cusps (or near the cusps) of the variation. The deviation is defined as the following equation:
Deviation $=\log$ (normalized measured value/ value calculated from the fitted curve).

Deviation patterns of dissolved REE in Fig. 2 demonstrate negative values at $\mathrm{Sm}$, Dy, Ho, Tm and $\mathrm{Yb}$, which is consistent with the variation of the W-type tetrad feature. The points of Eu are not counted because of its indigenous anomaly of crustal substances. It seems that negative deviations are more conspicuous in the HREE region than in the LREE region. It is not clear from Fig. 3 , however, which type of the effect the particu- 

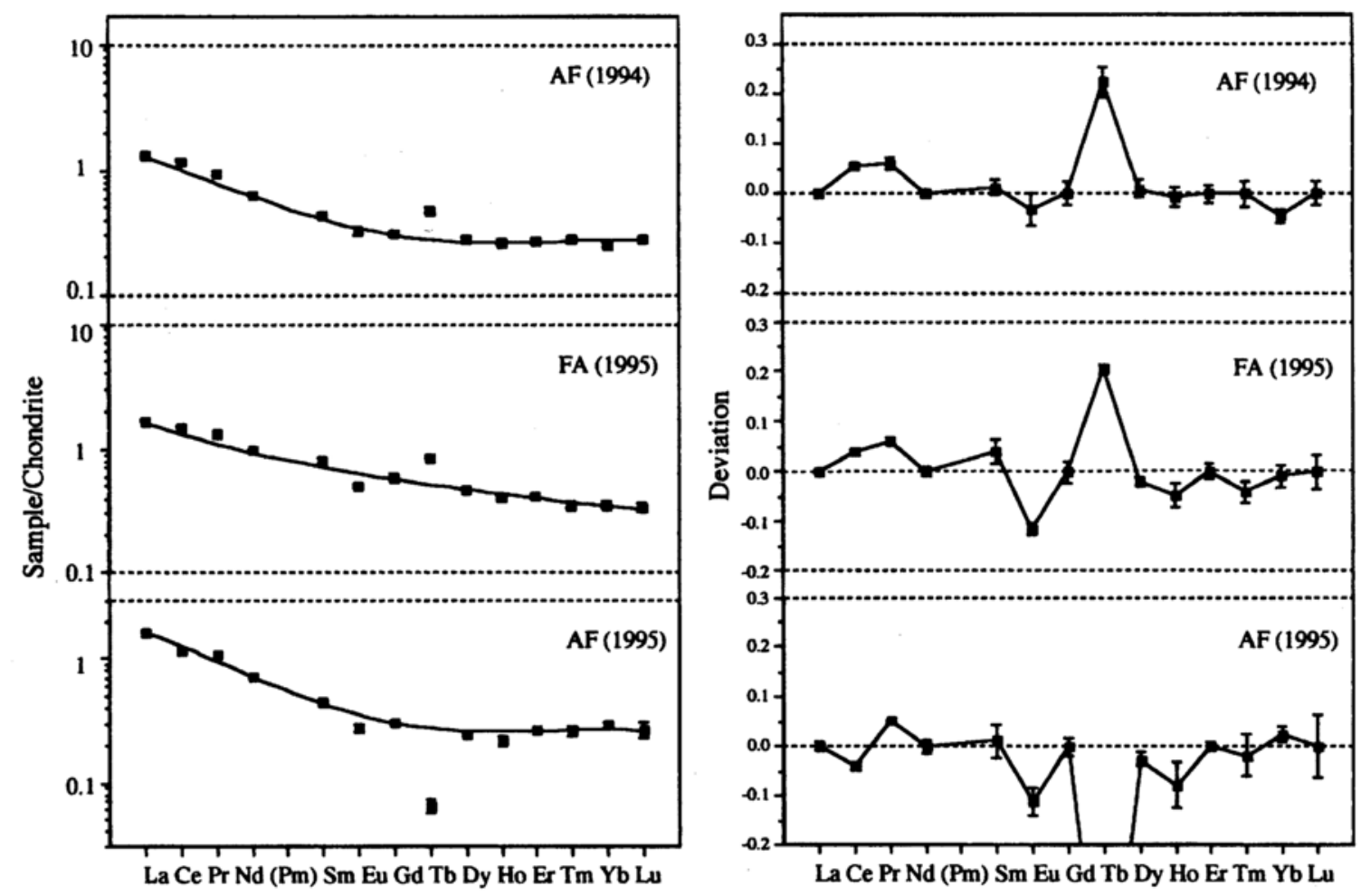

Fig. 3. Chondrite normalized patterns of particulate REE and deviations from fitting 4th dimensional curves.

late REE shows; the LREE region seems to show faint positive deviations except for $\mathrm{Ce}$ and $\mathrm{Eu}$, but no appreciable trend in the HREE region, being shrouded by greater error bars in this region. The amount of HREE in the sample is much smaller than those of LREE in collected and measurement of HREE with similar precision to LREE is quite difficult.

\section{REE partitioning between dissolved and particu- late forms}

The apparent partition coefficients of REE between dissolved and particulate forms were calculated using data in Table 2. In Fig. 4 the ratios are plotted on logarithmic scale against the atomic number of lanthanide with error bars $(1 \sigma)$ of the measurements. The "collected" of sample FA (1994) was not analyzed. The general trend of the partitioning pattern well reflects solubility of REE, which increases with their atomic numbers. Each deviation pattern of sample FA shows negative values at $\mathrm{Pr}, \mathrm{Sm}, \mathrm{Eu}, \mathrm{Tb}, \mathrm{Dy}, \mathrm{Ho}, \mathrm{Tm}$ and $\mathrm{Yb}$, suggesting the operation of the tetrad effect. Although sizes of error bars are quite significant, the variations demonstrated in Fig. 2 have become more regular in Fig. 4 with respect to the tetrad effect variation. (Anomalous plot of Tb in Fig. 2 is somewhat subdued and close to the trend of the tetrad effect.) The anomalies of Eu in Fig. 2 disappeared in Fig. 4, indicating that Eu behaves coherently with other REE and that the source of $\mathrm{Eu}$ anomaly of seawater is crustal particles which indigenously have Eu negative anomalies.

In the case of the sample-1995, the ratios show clear difference between the sample AF and FA; the negative deviation is more extensive in the sample FA than in the sample AF. This difference is due to the different contribution of the labile fraction in the analyses as indicated in Fig. 1, and implies that the labile fraction should show the $\mathbf{M}$ 

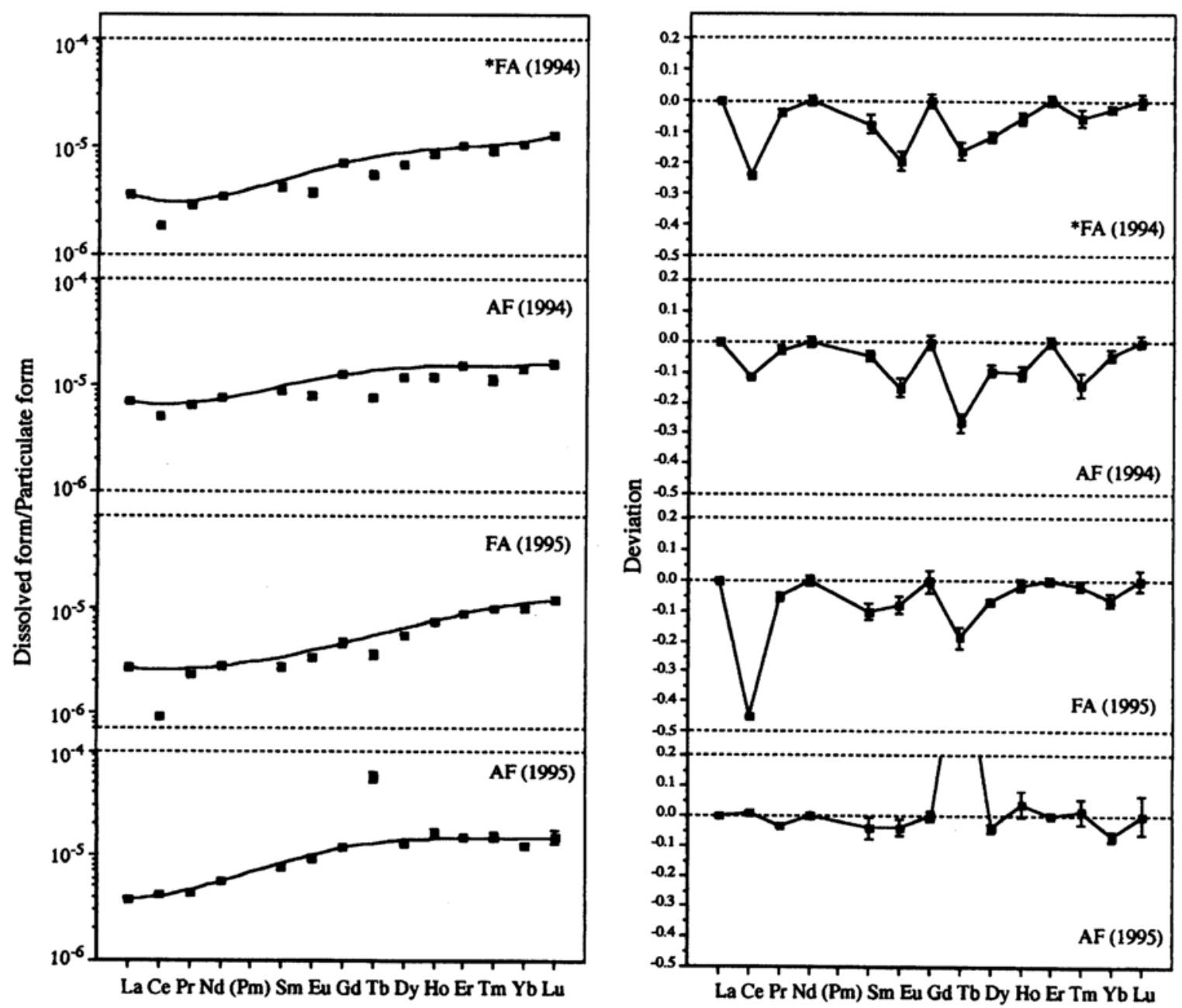

Fig. 4. Apparent REE partitioning patterns between dissolved and particulate fractions. *The data of "collected" of sample AF (1994) were used as that of sample FA (1994), since "collected" of the sample FA (1994) was not analyzed.

type tetrad effect. The similar difference can not be observed in the ratios of sample-1994. We did not measured the collected of the sample FA in 1994, making the discussion a little difficult unfortunately. Ce anomaly and $\mathrm{Tb}$ anomaly still appear in Fig. 4 and the reasons will be discussed later.

The distribution of metals between seawater and suspended particles has been expressed in terms of solution and surface chemical complexation (e.g., Balistrieri et al., 1981; Byrne and Kim, 1990):

$$
\frac{M_{T}}{M_{s}}=\frac{\Sigma_{n j}\left(1+{ }_{j}^{M} \beta_{n}\left[L_{j}\right]^{n}\right)}{\Sigma_{i}\left({ }_{i}^{M} K \cdot{ }_{s} L_{i}\right)},
$$

where $M_{T}$ is the total dissolved concentration of a given metal in seawater, $M_{s}$ is the concentration of metal bound to particulate matter in seawater, $\left[L_{j}\right]$ and ${ }_{s} L_{i}$ are dissolved and particulate ligand concentrations in seawater, and $j^{M} \beta_{n}$ and ${ }_{i}{ }^{M} K$ are equilibrium constants for the formation of dissolved metal complexes and surface-bound metal.

Byrne and Kim (1990) calculated the variation 


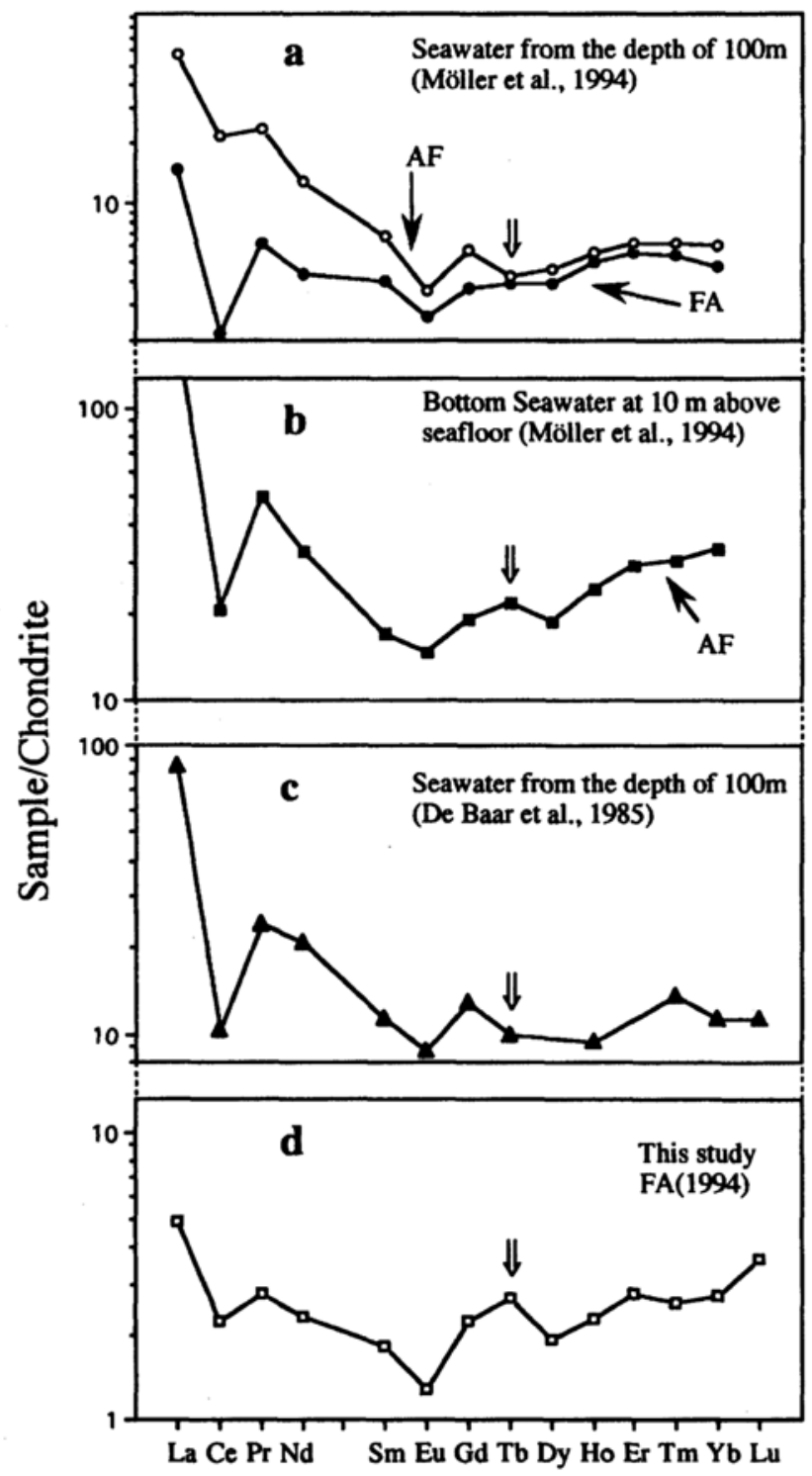

Fig. 5. Chondrite normalized patterns of dissolved REE in seawaters. a and b; by chelex preconcentration and ICP-MS, data from Möller et al. (1994). c; by chelex preconcentration and NAA, data from De Baar et al. (1985a).

of partitioning coefficients of REE in seawater using a variety of surface-types, and indicated that LREE are preferentially adsorbed compared to HREE. The affinity of REE for surface can be strongly affected by thin organic coatings, most representatively carboxylate, on suspended particles. Their model of REE distributions between seawater and suspended particles exhibit a strong dependence on $\mathrm{pH}$ and carbonate complexation.
According to their results, the REE patterns of distribution between seawater and suspended particles with carboxylate-rich surface at $\mathrm{pH} 7.9$ and 7.6 (Fig. 7 in their paper) are very similar to the apparent distribution ratios shown in Fig. 4 with respect to LREE/HREE ratio and "W type tetrad effect"-like variation. This remarkable consistency implies that REE pattern is influenced by organicrich surface of the particles in coastal area. 


\section{Tetrad effect}

As is stated in the introduction, the tetrad effect is the matter of debate (McLennan, 1994). We consider that the finding of two conjugate phases, each of which shows the $\mathrm{W}$ type and $\mathrm{M}$ type effect, respectively, and both of which are actually interacting with each other at the moment, would be one of the most convincing facts.

Most examples of the tetrad effect reported previously show that aqueous or dissolved phase has W type and solid phase has the M type with only few exceptions (e.g., Masuda et al., 1987). From the results of the previous works we presum that dissolved REE has $\mathrm{W}$ type and particulate REE does $M$ type.

The "W-type tetrad effect"-like variation seen in the ratio of filtrate/collected is consistent with the previous reports mentioned above. Since the abundance patterns of REE dissolved in seawater are the result of interaction with suspended solid, it is suggested that the tetrad effect observed in REE abundance in seawater is caused by the water and rock interaction. However the chemical mechanism causing the effect is still open to discussion. The most acceptable explanation for the effect is the configuration change of $4 \mathrm{f}$ electron during the chemical reaction (Jørgensen, 1970; Kawabe, 1992), but the REE patterns predicted by this theory is not identical to those of natural samples and further investigation is required for the study of tetrad effect.

\section{$\mathrm{Ce}$ and $\mathrm{Tb}$ anomalies}

The filtrates of the sample FA show a greater negative $\mathrm{Ce}$ anomaly than that of the sample AF, which indicates the selective incorporation of $\mathrm{Ce}$ in labile faction of particles and the incoherent behavior of $\mathrm{Ce}$ as $\mathrm{Ce}(\mathrm{IV})$. Oxidation potential of $\mathrm{Ce}(\mathrm{IV})$ is smaller than the other REE and in natural environment only $\mathrm{Ce}$ is known to have valency of +4 , whereas the other REE have $+3 . \mathrm{CeO}_{2}$, the unique species of $\mathrm{Ce}(\mathrm{IV})$, is hardly soluble and therefore solubility of $\mathrm{Ce}$ (IV) is much smaller than $\mathrm{Ce}(\mathrm{III})$ in aqueous medium.

Redox chemistry of Ce has been extensively studied by many researchers. Sunda et al. (1983) suggested photooxidation of Ce in surface water, and microbial processes were proposed as well (Moffet, 1994). Sholkovitz et al. (1994) indicated that the oxidation of $\mathrm{Ce}$ (III) is directly coupled to the redox chemistry of $\mathrm{Mn}$ in particulate $\mathrm{Mn}$ oxyhydroxide. A Mn oxide coated layer on the surface of particles collected in Tokyo Bay was reported by one of the present authors (Akagi and Haraguchi, 1994) and the mechanism reported by Sholkovitz et al. (1994) is quite feasible.

As mentioned several times in the previous sections, Tb anomaly was observed in several samples and also in the ratio between particulate and dissolved REE. We were perplexed by the capricious behavior of $\mathrm{Tb}$; in some REE patterns of concentration ratios between suspend particles and seawater $\mathrm{Tb}$ anomalies were seen, but in another not. In addition, we observed in the analyses of the same bottle of sample, some batches of particles showed positive anomaly, but other showed negative or no anomaly. Such capricious behavior of $\mathrm{Tb}$ makes us suspicious of the accuracy of our ICP-MS analysis. On the other hand, there are some facts of analytical chemistry which seem to support our results; the small and reproducible blank and constantly quantitative recovery of Tb. Another assuring fact is that the filtrate of sample AF always showed no Tb anomaly, and this type of sample (acidified before filtrated) is investigated by Shabani et al. (1992) and Zhang and Nozaki (1996), whose data gave no Tb anomaly. Scrutiny of the $\mathrm{Tb}$ data from different papers shows the difficulty of $\mathrm{Tb}$ measurements. Möller et al. (1994) have determined REE in seawaters of two types of samples (FA and AF) like those determined in our experiments, using ICP-MS. From their data, the inconsistency of Tb data between samples FA and AF can be observed in seawater from $100 \mathrm{~m}$ depth (Fig. 5a), and the positive $\mathrm{Tb}$ anomaly also can be observed in seawater (AF) at $10 \mathrm{~m}$ above seafloor (Fig. 5b) (note that $\mathrm{Lu}$ data were not reported). De Baar et al. $(1985 \mathrm{a}, \mathrm{b})$ filtrated seawater before acidified (FA) and reported a $\mathrm{Gd} / \mathrm{Tb}$ anomaly (Fig. 5c). They used neutron activation analysis instead of ICPMS to determine REE. We now have an impres- 
sion that $\mathrm{Tb}$, which has the second smallest oxidation potential, could be oxidized during any one of pretreatment procedures or storage. Kawabe (personal communication) experienced the concentration decrease of an acidified $\mathrm{Tb}$ solution during one year storage, which seems to imply the precipitation of $\mathrm{Tb}(\mathrm{IV})$. Further investigation is required to conclude the $\mathrm{Tb}$ anomaly in seawater.

\section{Conclusions}

1. "Tetrad effect"-like variation was partially observed in the REE pattern of dissolved and particulate REE, with ambiguous incoherency between LREE and HREE.

2. REE patterns of partitioning between dissolved and particulate REE show "W-type tetrad effect"-like variation. The "labile" fraction of REE on the particles is thought to have "M-type tetrad effect"-like variation, thus this fraction can be responsible for the appearance of "W-type tetrad effect"-like variation in seawater as the result of water-surface interaction.

3. Ce anomaly can be found in the REE patterns of partitioning between dissolved and particulate REE, Tb anomaly was found in the REE pattern of dissolved REE in sample FA, and capricious behavior of $\mathrm{Tb}$ for particles was observed in our experiments.

Acknowledgments-We thank staffs in Misaki Marine Biological Station, School of Science, University of Tokyo for helping us in off-shore sampling and for letting us use its convenient facilities.

\section{REFERENCES}

Akagi, T. and Haraguchi, H. (1994) The surface of particles in Tamagawa estuary and Tokyo Bay and their influence on the distribution of trace elements. Geochem. J. 28, 81-97.

Akagi, T., Shabani, M. B. and Masuda, A. (1993) Lanthanide Tetrad effect in Kimuraite $\left[\mathrm{CaY}_{2}\left(\mathrm{CO}_{3}\right)_{4} \cdot 6 \mathrm{H}_{2} \mathrm{O}\right]$; Implication of a new Geochemical Index. Geochim. Cosmochim. Acta 57, 2899-2905.

Balistrieri, L., Brewer, P. G. and Murray, J. W. (1981)
Scavenging residence times of trace metals and surface chemistry of sinking particles in the deep ocean. Deep-Sea Res. 28A, 101-110.

Byrne, R. H. and Kim, K.-H. (1990) Rare earth element scavenging in seawater. Geochim. Cosmochim. Acta 54, 2645-2656.

De Baar, H. J. W., Bacon, M. P. and Brewer, P. G. (1985a) Rare earth elements in the Pacific and Atlantic Oceans. Geochim. Cosmochim. Acta 49, 19431959.

De Baar, H. J. W., Bacon, M. P. and Brewer, P. G. (1985b) Anomalies in rare earth distributions in seawater: Gd and Tb. Geochim. Cosmochim. Acta 49, 1961-1969.

Elderfield, H. and Greaves, M. J. (1982) The rare earth elements in seawater. Nature 296, 214-219.

Henderson, P. (1984) REE Geochemistry. Chap. 5, Rare earth element abundances in upper mantle rocks, 153-196, Elsevier.

Jørgensen, C. K. (1970) The "tetrad effect" of Peppard is a variation of the nephelauxetic ratio in the third decimal. J. Inorg. Nucl. Chem. 32, 3127-3128.

Kawabe, I. (1992) Lanthanide tetrad effect in the $\mathrm{Ln}^{3+}$ ionic radii and refined spin-pairing energy theory. Geochem. J. 26, 309-335.

Klinkhammer, G., Elderfield, H. and Hudson, A. (1983) Rare earth elements in seawater near hydrothermal vents. Nature 305, 185-188.

Koeppenkastrop, D. and De Carlo, E. H. (1993) Uptake of rare earth elements from solutions by metal oxides. Environ. Sci. Technol. 27, 1796-1802.

Koeppenkastrop, D., De Carlo, E. H. and Roth, M. (1991) A method to investigate the interaction of rare earth elements in aqueous solution with metal oxides. J. Radioanal. Nucl. Chem. 152, 337-346.

Masuda, A. (1975) Abundances of monoisotopic REE, consistent with Leedey chondrite values. Geochem. J. 9, 183-184.

Masuda, A. and Ikeuchi, Y. (1979) Lanthanide tetrad effect observed in marine environment. Geochem. J. 13, 19-22.

Masuda, A., Nakamura, N. and Tanaka, T. (1973) Fine structures of mutually normalized rare earth patterns of chondrites. Geochim. Cosmochim. Acta 37, 239248.

Masuda, A., Kawakami, O., Dohmoto, Y. and Takenaka, T. (1987) Lanthanite tetrad effects in nature: two mutually opposite types, $\mathrm{W}$ and $\mathrm{M}$. Geochem. J. 21, 119-124.

McLennan, S. M. (1994) Rare earth element geochemistry and the "tetrad" effect. Geochim. Cosmochim. Acta 58, 2025-2033.

Moffett, J. W. (1994) The relationship between Ce and Mn oxidation in the marine environment. Limnol. 
Oceanogr. 39, 1309-1318.

Möller, P., Dulski, P. and Bau, M. (1994) Rare-Earth Element adsorption in a Seawater Profile above the East Pacific Rise. Chem. Erde 54, 129-149.

Peppard, D. F., Mason, G. W. and Lewey, S. (1969) A tetrad effect in the liquid-liquid extraction ordering of lanthanides(III). J. Inorg. Nucl. Chem. 31, 22712272.

Piepgras, D. J. and Jacobsen, S. B. (1992) The behavior of rare earth elements in seawater: precise determination of variations in the North Pacific water column. Geochim. Cosmochim. Acta 56, 1851-1862.

Shabani, M. B., Akagi, T. and Masuda, A. (1992) Preconcentration of trace rare earth elements in seawater by complexation with bis (2-ethylhexyl) hydrogen phosphate and 2-ethylhexyl dihydrogen phosphate adsorbed on a $\mathrm{C}_{18}$ cartridge and determination by inductively coupled plasma mass spectrometry. Anal. Chem. 64, 737-743.

Sholkovitz, E. R., William, M. A. and Brent, L. L. (1994) Ocean particle chemistry: The fractionation of rare earth elements between suspend particles and seawater. Geochim. Cosmochim. Acta 58, 1567-1579.

Sunda, W. G., Huntsman, S. A. and Harvey, G. R. (1983) Photoreduction of manganese oxides in seawater and its geochemical and biological implications. Nature 301, 234-236.

Zhang, J. and Nozaki, Y. (1996) Rare earth elements and yttrium in seawater: ICP-MS determinations in the East Caroline, Coral Sea, and South Fiji basins of the western South Pacific Ocean. Geochim. Cosmochim. Acta 60, 4631-4644. 